\title{
Nonlinear Ellipsoid Based Attitude Control for Aggressive Trajectories in a Quadrotor: Closed-Loop Multi-Flips Implementation
}

\author{
Fatima Oliva-Palomo ${ }^{\mathrm{a}, *}$, Anand Sanchez-Orta $^{\mathrm{a}}$, Pedro Castillo $^{\mathrm{b}}$, Hussain Alazki $^{\mathrm{c}}$ \\ ${ }^{a}$ Robotics and Advanced Manufacturing Division, Research Center for Advanced Studies (Cinvestav), \\ Saltillo México. \\ ${ }^{b}$ Sorbonne Universités, Université de Technologie de Compiègne, CNRS UMR 7253 Heudiasyc Lab., CS \\ 60319, 60203 Compiègne Cedex, France. \\ ${ }^{c}$ Department of Mechatronics, Autonomous University of Carmen (UNACAR), Mexico.
}

\begin{abstract}
In this paper a model-free continuous nonlinear control law for the attitude of a quadrotor, based on the Attractive Ellipsoid Method and a saturation term, is proposed. This control law allows the vehicle to track aggressive maneuvers, such as multiple flips about the $y$ axis of the body frame, with high angular velocities. The controller is designed through a singularity-free attitude representation based on a unit quaternion and its gains are computed by solving an optimization problem with LMIs. The proposed controller preserves the advantageous characteristics of the Attractive Ellipsoid Method and increases its robustness properties with the fast response of the nonlinear saturation term, minimizing as much as possible the attitude tracking error and assuring its convergence to a small neighborhood around the origin. A numerical study based on simulations is presented to analyze the advantages of the proposed approach, and experiments are presented to show the performance of the closed-loop system for tracking aggressive multiple flips, even in outdoors.

Keywords: Attitude control, Attractive Ellipsoid Method, Quadrotor UAV, Aggressive Maneuvers, Real-time validation, Quaternion approach.
\end{abstract}

\section{Introduction}

The quadrotors are fast light weight vehicles that present high maneuverability to perform many different trajectories, making them attractive for diverse applications, including their use as experimental platforms for scientific purposes. Nowadays, it is considered that their autonomy level is enough to accomplish high-level control task.

\footnotetext{
${ }^{*}$ Corresponding author

Email addresses: fatima.oliva@cinvestav.mx (Fatima Oliva-Palomo), anand. sanchez@cinvestav.mx (Anand Sanchez-Orta), pedro.castillo@hds.utc.fr (Pedro Castillo), halazki @pampano. unacar.mx (Hussain Alazki)
} 
However, some challenging tasks, such as physical interaction of quadrotors or trajectory tracking at high frequencies, require the full quadrotor's dynamic model as well as uncertainties and disturbances to be considered in the control design, which cannot be handled by approaches based on either linearization, the direct compensation of nonlinear terms or the assumption of quasi-hovering flight. In addition, these vehicles are subject to many aerodynamic effects that cannot be fully modeled or directly compensated in the control law, making them prone to oscillations and thus compromising stability.

In order to assure tracking of aggressive maneuvers a robust control approach is needed to attenuate the effect of endogenous and exogenous disturbances present in the quadrotor dynamics, [1,2]. Also, the correct choice in the attitude representation is important because there are some representations that are not well defined for some points, causing the desired trajectories to be constrained by assumption to trajectories sufficiently close to the origin, which physically cannot prevent the system from reaching singular points. Several research efforts have been conducted for the control of the quadrotor's attitude dynamics. Some of these researches focus on global controllers to avoid singularities in the attitude representation by using unit quaternions $[3,4,5]$. However, it is important to notice that there is a sign ambiguity when unit quaternions are used to represent the attitude of the quadrotor, which is due to the fact that a given unit quaternion and its negative represent the same rotation. This ambiguity can be avoided either by considering other attitude representations [6] or by using a signal conditioning stage (which is the case in our proposed approach).

The control design for quadrotors performing aggressive maneuvers has been treated in $[7,8,9]$, and, specifically, the execution of multi-flips maneuvers has been considered in $[10,11,12,13]$. The proposed scheme in [10] is based on a bang-bang-like control strategy with a learning process that requires a large number of iterations. At each iteration the maneuver (a number of flips) is performed and a state error is used to update the control parameters. This state error is measured by a Vicon motion capture system at $200 \mathrm{~Hz}$ with millimeter accuracy. In addition, the quadrotor performs the flips by setting the maximum differential thrust to achieve a maximum desired angular velocity. Schemes of this kind can be successful in controlled environments, however, in outdoors the performance of the tracking gets worse if an external disturbance is present while the vehicle executes the maneuver. In addition, when aerodynamic disturbances, generated by the rotors, motion of the vehicle or the environment, are not considered $[3,12,7,9,5]$, the tracking trajectory may not be assured. Although some approaches consider disturbances with a closed-loop control design $[4,8,14]$, their attitude representation is not well defined for every point, restricting the maneuvers that the vehicle can accomplish. Furthermore, robust discontinuous control strategies that compensate theoretically disturbances present in the system, usually based on sliding modes, induce oscillations on the vehicle $[14,1]$, and thus instability, even if the sign function is approximated by high frequency smooth functions.

In this paper, inspired by the Attractive Ellipsoid Method [15, 16], a continuous nonlinear attitude controller for the tracking of aggressive maneuvers is proposed. The proposed control uses the combination of the Ellipsoid method and a saturation term applied to a quadrotor to design a robust attitude scheme free of singularities, ensuring a continuous yet robust controller to enforce practical stability of tracking errors, even 
if the required trajectory is aggressive. The controller is composed of two terms: a state feedback, which induces an attractive invariant set around the origin, and a nonlinear saturation element, which improves robustness as well as frequency response without significantly modifying the magnitude of the controller, minimizing the effects of uncertainties and disturbances in such a way that the upper bound of the invariant set is reduced. In our approach a unit-quaternion representation is used to avoid singularities that other representations have, like Euler angles. The controller gains are established by solving an optimization problem, where Linear Matrix Inequalities (LMIs) appear. These LMIs are numerically solved offline to minimize as much as possible the attitude tracking error, assuring its convergence to a small neighborhood around the origin (practical stability). The resulting controller is independent of the parameters and dynamical structure of the system (model free), and generates continuous signals with frequencies that can be handled by the rotors. Thus, it preserves the advantageous characteristics of the Attractive Ellipsoid Method and increases its robustness properties with the fast response of the saturation term. The performance of the controller is demonstrated in experiments using as an aggressive desired trajectory multiple flips about the $y$ axis, which are executed even in outdoors.

The rest of this paper is organized as follows: the attitude dynamical model of the quadrotor and the necessary background to design the controller are presented in Section 2. Section 3 describes the control problem by declaring the goal of the controller and the assumptions for the stability analysis. Section 4 defines the attitude error and its dynamics. The control design and its stability analysis are presented in Section 5. Numerical simulations and the contribution of the saturation term are analyzed in Section 6. Section 7 shows the experimental results of indoor and outdoor multiple flips by describing the experimental setup and the operative conditions. Finally, the main conclusions are discussed in section 8 .

\section{Background}

In order to provide the required conditions and properties of the control design, the following definitions are presented.

\subsection{The Class of quasi-Lipschitz Functions}

Definition 1[17]: A vector function $g: \mathbb{R}^{n} \rightarrow \mathbb{R}^{k}$ is said to be from the class $C\left(\mathbf{C}, \delta_{0}, \delta_{1}\right)$ of quasi-Lipschitz functions if there exist matrix $\mathbf{C} \in \mathbb{R}^{k \times n}$ and nonnegative constants $\delta_{0}$ and $\delta_{1}$ such that for any $\mathbf{x} \in \mathbb{R}^{n}$ the following inequality holds:

$$
\|g(\mathbf{x})-\mathbf{C x}\|^{2} \leq \delta_{0}+\delta_{1}\|\mathbf{x}\|^{2}
$$

This implies that the growth rates of $g(\mathbf{x})$ as $\|\mathbf{x}\| \rightarrow \infty$ are not faster than linear, [16]. Notice that if $\mathbf{C}=0, \delta_{0}=0$ and $g(0)=0$ the inequality (1) characterizes the Lipschitz continuity property of the function $g(\mathbf{x})$ with the Lipschitz constant $L=\sqrt{\delta_{1}}$. 


\subsection{Attractive Ellipsoids}

Definition 2 [16]: An ellipsoid, which is represented as $\mathcal{E} \subset \mathbb{R}^{n}$ with center in $\mathbf{x}_{c}$ and given by:

$$
\mathcal{E}=\left\{\mathbf{x}(t) \in \mathbb{R}^{n} \mid\left(\mathbf{x}(t)-\mathbf{x}_{c}\right)^{\top} \mathbf{P}\left(\mathbf{x}(\mathbf{t})-\mathbf{x}_{\mathbf{c}}\right) \leq 1\right\}
$$

is said to be attractive for the closed-loop system $\dot{\mathbf{x}}(t)=g(t, \mathbf{x}(t), u(\mathbf{x}(t))), t \geq 0$, if:

$$
\limsup _{t \rightarrow \infty}\left(\mathbf{x}(\mathbf{t})-\mathbf{x}_{\mathbf{c}}\right)^{\top} \mathbf{P}\left(\mathbf{x}(\mathbf{t})-\mathbf{x}_{\mathbf{c}}\right) \leq 1
$$

where the ellipsoidal matrix $\mathbf{P}$ is a symmetric positive definite matrix $0<\mathbf{P}=\mathbf{P}^{\top} \epsilon$ $\mathbb{R}^{n \times n}$. Note that a inertia matrix $\mathbf{J}$ fulfills the requirements for a ellipsoidal matrix $0<\mathbf{J}=\mathbf{J}^{\top} \in \mathbb{R}^{3 \times 3}$. In particular, equation (2) represents the attractive invariant set that will be constructive for the stability analysis of the attitude error.

\subsection{Quadrotor Dynamical Model}

The forces that produce the motion of the quadrotor are generated by the rotors. Each rotor generates a thrust force and a reaction torque that result from the aerodynamic effects of the propeller rotation. These forces are roughly proportional to the squared angular velocities of the rotors and can be expressed as [3]:

$$
T_{i}=b \varpi_{i}^{2}, M_{i}=b l \varpi_{i}^{2}, Q_{i}=k \varpi_{i}^{2}
$$

where, $\varpi_{i}$ is the angular velocity of the rotor $i, T_{i}$ is the thrust generated by the rotor $i, M_{i}$ is the moment created by the thrust $T_{i}, Q_{i}$ is the reaction torque generated by the rotor $i, i \in\{1,2,3,4\}, l$ is the distance from each rotor to the center of mass of the vehicle, and $b, k$ are positive aerodynamic parameters. In order to balance the reaction torques, the four-rotor configuration requires that two of its propellers rotate in clockwise direction while the other two rotate in the opposite direction, as can be seen in Figure 1. The forces and moments produced by the rotors are arranged as a resultant thrust force $T \in \mathbb{R}$ and a torque $\tau \in \mathbb{R}^{3}$, which can be expressed in matrix form as

$$
\left[\begin{array}{c}
T \\
\tau_{x} \\
\tau_{y} \\
\tau_{z}
\end{array}\right]=\left[\begin{array}{cccc}
b & b & b & b \\
\frac{b l}{\sqrt{2}} & \frac{b l}{\sqrt{2}} & -\frac{b l}{\sqrt{2}} & -\frac{b l}{\sqrt{2}} \\
\frac{b l}{\sqrt{2}} & -\frac{b l}{\sqrt{2}} & -\frac{b l}{\sqrt{2}} & \frac{b l}{\sqrt{2}} \\
k & -k & k & -k
\end{array}\right]\left[\begin{array}{c}
\varpi_{1}^{2} \\
\varpi_{2}^{2} \\
\varpi_{3}^{2} \\
\varpi_{4}^{2}
\end{array}\right]
$$

where $\tau=\left[\tau_{x}, \tau_{y}, \tau_{z}\right]^{\top}$. The map (3) is invertible, then, $T$ and $\tau$ can be considered as the control inputs of the system that become desired angular velocities of the rotors through map (3) .

Now, the dynamical model of the quadrotor is derived by considering the torque $\tau$ as the control input. Let us define the earth fixed frame as $\mathcal{I}=\left\{\mathbf{e}_{x}, \mathbf{e}_{y}, \mathbf{e}_{z}\right\}$ and the body fixed frame be $\mathcal{B}=\left\{\mathbf{e}_{x}^{b}, \mathbf{e}_{y}^{b}, \mathbf{e}_{z}^{b}\right\}$, whose origin coincides with the center of mass. The Euler equations of motion representing the orientation dynamics of the quadrotor by using a unit quaternion to avoid singularities are defined as follows:

$$
\dot{\overline{\mathbf{q}}}=\frac{1}{2} \overline{\mathbf{q}} \otimes \overline{\boldsymbol{\omega}}
$$




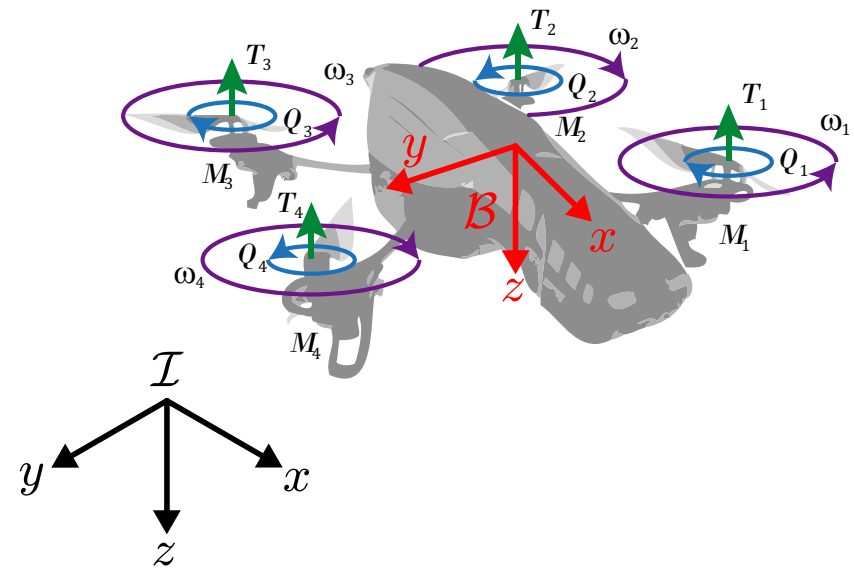

Figure 1: The Parrot AR. Drone, with the direction of rotations of propellers, forces, moments, inertial and body frames

$$
\mathbf{J} \dot{\omega}=-\omega \times \mathbf{J} \omega+\tau+\mathbf{d}(t)
$$

where, $\overline{\mathbf{q}}=\left[q_{0}, \mathbf{q}^{\top}\right]^{\top}, \overline{\mathbf{q}} \in S^{3}=\{\overline{\mathbf{q}} \in \mathbb{H}:\|\overline{\mathbf{q}}\|=1\}$ with $S^{3}$ the unit sphere in the space of quaternions, $\mathbb{H}=\left\{q_{0}, \mathbf{q}: q_{0} \in \mathbb{R}, \mathbf{q} \in \mathbb{R}^{3}\right\}$ is the set of quaternions, $\omega=\left[\omega_{x}, \omega_{y}, \omega_{z}\right]^{\top} \in \mathcal{B}$ defines the angular velocity of the airframe, $\bar{\omega}=\left[0, \omega^{\top}\right]^{\top}$ represents a pure quaternion and $\mathbf{J} \in \mathbb{R}^{3 \times 3}$ stands for the constant inertia matrix around the center of mass expressed in the body fixed frame $\mathcal{B}$. The external bounded torque disturbance is denoted by $\mathbf{d}(t)$, and $\tau \in \mathcal{B}$ is the control torque. The quaternion product is defined as:

$$
\overline{\mathbf{p}} \otimes \overline{\mathbf{q}}=\left[\begin{array}{c}
p_{0} q_{0}-\mathbf{p}^{\top} \mathbf{q} \\
p_{0} \mathbf{q}+q_{0} \mathbf{p}+[\mathbf{p} \times] \mathbf{q}
\end{array}\right]
$$

and matrix $[\boldsymbol{p} \times] \in \mathbb{R}^{3 \times 3}$ denotes the skew-symmetric matrix of the vector $\boldsymbol{p}$

$$
[\boldsymbol{p} \times]=\left[\begin{array}{ccc}
0 & -p_{3} & p_{2} \\
p_{3} & 0 & -p_{1} \\
-p_{2} & p_{1} & 0
\end{array}\right] .
$$

\section{Problem formulation}

Let us consider a user defined attitude trajectory denoted by the unit quaternion $\overline{\mathbf{q}}_{d}=\left[q_{0 d}, \mathbf{q}_{d}^{\top}\right]^{\top}$. In addition, for a given $\overline{\mathbf{q}}_{d}$ there exists a desired angular velocity $\omega_{d}(t) \in \mathcal{B}$ that satisfies the following differential equation:

$$
\dot{\overline{\mathbf{q}}}_{d}=\frac{1}{2} \overline{\mathbf{q}}_{d} \otimes \overline{\boldsymbol{\omega}}_{d}
$$

where $\bar{\omega}_{d}=\left[0, \omega_{d}^{\top}\right]^{\top}$. 
The problem is to design a closed-loop controller, which allows the quadrotor to track aggressive attitude trajectories, for the system (4)-(5), assuring that $\overline{\mathbf{q}} \rightarrow \overline{\mathbf{q}}_{d}$ and $\omega \rightarrow \omega_{d}$ (where their error trajectories are confined to a small region of an attractive set), independent of the parameters and dynamic structure of the system (model free), subject to model and parameter uncertainties, aerodynamic effects as well as exogenous disturbances, and able to generate continuous signals that can be handled by the rotors. The control design considers the following assumptions:

Assumption 1: The desired angular velocity $\omega_{d}(t)$ and its derivative $\dot{\omega}_{d}(t)$ are sufficiently smooth functions defined by the user and are bounded by

$$
\left\|\omega_{d}\right\|^{2} \leq \omega_{d}^{+} \quad\left\|\dot{\omega}_{d}\right\|^{2} \leq \dot{\omega}_{d}^{+}
$$

where $\omega_{d}^{+}$and $\dot{\omega}_{d}^{+}$are positive constants.

Assumption 2: The norm of the external disturbance $\mathbf{d}(t)$ has an upper limit, then a constant $d^{+}$exists such that

$$
\|\mathbf{d}(t)\|^{2}<d^{+}
$$

Assumption 3: The nonlinear term $-\boldsymbol{\omega} \times \mathbf{J} \omega$ in the dynamic system (5), can be bounded as:

$$
\|-\omega \times \mathbf{J} \omega\|^{2} \leq c_{0}+c_{1}\|\omega\|^{2} \forall \omega \in \mathbb{R}^{3}
$$

In addition to this main problem, we will also need to find the gains that guarantee the boundedness of the error trajectories by estimating an attractive ellipsoid in each operating condition of the control system. Now, let us define the attitude error and its dynamics in order to design the controller and analyze the stability of the closed-loop system.

\section{Error Dynamics}

\subsection{Error definitions}

Consider $\overline{\mathbf{q}}_{e}$ as the error quaternion that represents a rotation between the real and the desired attitude, given by

$$
\overline{\mathbf{q}}_{e}=\overline{\mathbf{q}}_{d}^{*} \otimes \overline{\mathbf{q}}
$$

where $\overline{\mathbf{q}}_{d}^{*}=\left[q_{0 d},-\mathbf{q}_{d}^{\top}\right]^{\top}$ is the conjugate of the desired quaternion, which corresponds to the inverse for unit quaternions.

Defining the angular velocity error as $\omega_{e}=\omega-\omega_{d}$, and using the equations (4), (7) and (8), the relation between the quaternion error and the angular velocity error can be obtained

$$
\dot{\mathbf{q}}_{e}=\frac{1}{2}\left[\begin{array}{c}
-\mathbf{q}_{e}^{\top} \omega_{e} \\
\left(q_{0 e} \mathbf{I}+\left[\mathbf{q}_{e} \times\right]\right) \omega_{e}+2 \mathbf{q}_{e} \times \omega_{d}
\end{array}\right]
$$

Now, define the error manifold as

$$
\mathbf{s}_{r}=\mathbf{C x}=\omega_{e}+\alpha \mathbf{q}_{e}
$$


where $\alpha$ is a positive-definite diagonal matrix, and

$$
\mathbf{C}=\left[\begin{array}{ll}
\alpha & \mathbf{I}
\end{array}\right], \quad \mathbf{x}=\left[\mathbf{q}_{e}^{\top} \boldsymbol{\omega}_{e}^{\top}\right]^{\top}
$$

Now, the main objective is to assure that the error manifold $\mathbf{s}_{r} \rightarrow 0$, which implies that $\omega_{e} \rightarrow 0$ and $\mathbf{q}_{e} \rightarrow 0$. It is worth noting that an error manifold is defined as $s=\dot{x}+\alpha x$ in general, however, $\mathbf{s}_{r}$ defined in (10) is not a homogeneous linear equation as a result of $\dot{\mathbf{q}}_{e} \neq \omega_{e}$. Nevertheless, this issue can be solved by considering that $\dot{\mathbf{q}}_{e} \rightarrow-\frac{\alpha}{2} q_{0 e} \mathbf{q}_{e}$ when $\omega_{e} \rightarrow \alpha \mathbf{q}_{e}$, [18]. The next step is to define the considered uncertainties and disturbances through the dynamics of the error manifold, which represents the openloop error dynamics.

\subsection{Open-Loop Error Dynamic Equation}

Taking the time derivative of $\mathbf{s}_{r}$ in (10), and using (9) it follows that

$$
\dot{\mathbf{s}}_{r}=\dot{\omega}-\dot{\omega}_{d}+\frac{1}{2} \boldsymbol{\alpha}\left(q_{0 e} \mathbf{I}+\left[\mathbf{q}_{e} \times\right]\right) \omega_{e}-\alpha\left[\omega_{d} \times\right] \mathbf{q}_{e}
$$

multiplying the equation (12) by the inertia matrix it yields

$$
\mathbf{J} \dot{\mathbf{s}}_{r}=\tau+\zeta\left(\omega, \omega_{d}, \dot{\omega}_{d}, \overline{\mathbf{q}}_{e}, t\right)
$$

where

$$
\begin{aligned}
\zeta\left(\omega, \omega_{d}, \dot{\omega}_{d}, \overline{\mathbf{q}}_{e}, t\right)= & -\omega \times \mathbf{J} \omega+\mathbf{d}(t)-\mathbf{J} \dot{\omega}_{d}-\mathbf{J} \alpha\left[\omega_{d} \times\right] \mathbf{q}_{e} \\
& +\frac{1}{2} \mathbf{J} \alpha\left(q_{0 e} \mathbf{I}+\left[\mathbf{q}_{e} \times\right]\right) \omega_{e}
\end{aligned}
$$

\subsection{Upper bound of $\zeta$}

In this subsection, an upper bound of $\zeta$ is derived. Note that $\zeta$ depends on both $\omega$ and $\omega_{e}$, consequently, their upper bounds are also needed. To this end, the following extended vector and its properties are introduced.

Consider $\tilde{\mathbf{x}}=\left[\mathbf{q}_{e}^{\top} \omega_{e}^{\top} \omega_{d}^{\top}\right]^{\top}$, with the following equalities

$$
\begin{aligned}
\left\|\omega_{e}\right\|^{2}=\tilde{\mathbf{x}}^{\top} \mathbf{D} \tilde{\mathbf{x}}, \quad\|\boldsymbol{\omega}\|^{2}=\tilde{\mathbf{x}}^{\top} \mathbf{E}^{\top} \mathbf{E} \tilde{\mathbf{x}}, \quad \mathbf{q}_{e}=\mathbf{F} \tilde{\mathbf{x}}, \\
\mathbf{x}=\mathbf{G} \tilde{\mathbf{x}}, \quad \mathbf{s}_{r}=\tilde{\mathbf{G}} \tilde{\mathbf{x}}
\end{aligned}
$$

with

$$
\begin{gathered}
\mathbf{D}=\left[\begin{array}{lll}
0 & 0 & 0 \\
0 & \mathbf{I} & 0 \\
0 & 0 & 0
\end{array}\right], \mathbf{E}=\left[\begin{array}{lll}
0 & \mathbf{I} & \mathbf{I}
\end{array}\right], \mathbf{F}=\left[\begin{array}{lll}
\mathbf{I} & 0 & 0
\end{array}\right], \\
\mathbf{G}=\left[\begin{array}{lll}
\mathbf{I} & 0 & 0 \\
0 & \mathbf{I} & 0
\end{array}\right], \tilde{\mathbf{G}}=\mathbf{C G}
\end{gathered}
$$

From (10), $\omega_{e}$ can be expressed as

$$
\omega_{e}=-\alpha \mathbf{q}_{e}+\mathbf{s}_{r}
$$

Then, using (15) a bound of (16) can be given by

$$
\left\|\omega_{e}\right\|^{2} \leq\left\|\mathbf{s}_{r}\right\|^{2}+\lambda_{\max }(\boldsymbol{\alpha})^{2}-\tilde{\mathbf{x}}^{\top} \tilde{\mathbf{F}} \tilde{\mathbf{x}}
$$


where $\tilde{\mathbf{F}}=\tilde{\mathbf{G}}^{\top} \boldsymbol{\alpha} \mathbf{F}+\mathbf{F}^{\top} \boldsymbol{\alpha} \tilde{\mathbf{G}}$ and $\lambda_{\max }(\boldsymbol{\alpha})$ is the maximum eigenvalue of $\boldsymbol{\alpha}$.

Observe that $\left\|\mathbf{q}_{e}\right\| \leq 1,\left|q_{0 e}\right| \leq 1,0<j^{-}<\lambda_{\min }(\mathbf{J})<\|\mathbf{J}\|<\lambda_{\max }(\mathbf{J})<j^{+}$, where $\lambda_{\min }(\mathbf{J})$ is the minimum eigenvalue of $\mathbf{J}$. Thus, using the assumptions of section 3 , the bound of (14) can be computed as

$$
\begin{aligned}
\|\zeta\|^{2}= & \zeta\left(\omega, \omega_{d}, \dot{\omega}_{d}, \overline{\mathbf{q}}_{e}, t\right)^{\top} \zeta\left(\omega, \omega_{d}, \dot{\boldsymbol{\omega}}_{d}, \overline{\mathbf{q}}_{e}, t\right) \\
\leq & 5\left(c_{0}+c_{1}\|\omega\|^{2}+d^{+}+\|\mathbf{J}\|^{2} \dot{\omega}_{d}^{+}\right. \\
& \left.+\|\mathbf{J}\|^{2}\|\boldsymbol{\alpha}\|^{2} \omega_{d}^{+}+\frac{1}{4}\|\mathbf{J}\|^{2}\|\boldsymbol{\alpha}\|^{2}\left\|\boldsymbol{\omega}_{e}\right\|^{2}\right) \\
\leq & c_{2}+c_{3}\|\boldsymbol{\omega}\|^{2}+c_{4}\left\|\boldsymbol{\omega}_{e}\right\|^{2} \\
\leq & c_{2}+\tilde{x}^{\top} \mathbf{H} \tilde{x}
\end{aligned}
$$

where $c_{2}=5 c_{0}+5 d^{+}+5 \lambda_{\max }(\mathbf{J})^{2} \lambda_{\max }(\boldsymbol{\alpha})^{2} \omega_{d}^{+}+5 \lambda_{\max }(\mathbf{J})^{2} \dot{\omega}_{d}^{+}, c_{3}=5 c_{1}, c_{4}=\frac{5}{4} \lambda_{\max }(\mathbf{J})^{2} \lambda_{\max }(\boldsymbol{\alpha})^{2}, \mathbf{H}=$ $c_{4} \mathbf{D}+c_{3} \mathbf{E}^{\top} \mathbf{E}$.

\section{Main Result}

Consider the following control law

$$
\boldsymbol{\tau}=-\mathbf{K}_{d} \mathbf{s}_{r}-\beta \operatorname{sat}\left(\gamma \mathbf{s}_{r}\right)
$$

where $\mathbf{K}_{d}$ and $\gamma$ are positive-definite diagonal matrices, $\beta$ is a positive constant, the saturation function is defined by $\operatorname{sat}\left(\gamma \mathbf{s}_{r}\right)=\left[\operatorname{sat}\left(\gamma_{1} s_{r 1}\right), \operatorname{sat}\left(\gamma_{2} s_{r 2}\right), \operatorname{sat}\left(\gamma_{3} s_{r 3}\right)\right]^{\top}$ and

$$
\operatorname{sat}\left(\gamma_{i} s_{r i}\right)= \begin{cases}-1 & \text { if } \gamma_{i} s_{r i} \leq-1 \\ \gamma_{i} s_{r i} & \text { if }-1<\gamma_{i} s_{r i}<1 \\ 1 & \text { if } \gamma_{i} s_{r i} \geq 1\end{cases}
$$

$\forall i \in\{1,2,3\}$.

The main idea behind the control law (19) is the following: the term $\mathbf{K}_{d} \mathbf{s}_{r}$ is used to induce an attractive invariant set around the origin, while the term $\beta \operatorname{sat}\left(\gamma \mathbf{s}_{r}\right)$ is introduced to minimize the effects of uncertainties and disturbances present in the system (13).

Now, the main result is stated in the following theorem.

Theorem 1. Suppose that

1.- The assumptions 1-3 are fulfilled;

2.- For the given matrix $\mathbf{J}$ there exists a matrix $\mathbf{K}_{d}$, and positive constants $\alpha_{\varepsilon}, \varepsilon_{1}, \varepsilon_{2}$, $\varepsilon_{3}$, such that the matrix $\mathbf{W}_{0}\left(\mathbf{K}_{p}, \mathbf{J}, \alpha_{\varepsilon}, \varepsilon_{1}, \varepsilon_{2}, \varepsilon_{3}\right)$ is negative semidefinite, where:

$$
\begin{gathered}
\mathbf{W}_{0}=\left[\begin{array}{cc}
\tilde{\mathbf{G}}^{\top}\left(-\mathbf{K}_{d}-\mathbf{K}_{d}^{\top}+\alpha_{\varepsilon} \mathbf{J}+\varepsilon_{2} \mathbf{I}\right) \tilde{\mathbf{G}} & \tilde{\mathbf{G}}^{\top} \\
+\varepsilon_{1} \mathbf{H}-\tilde{\mathbf{H}}-\varepsilon_{2} \tilde{\mathbf{F}} & \\
\tilde{\mathbf{G}} & -\varepsilon_{1} \mathbf{I}
\end{array}\right] \\
\tilde{\mathbf{H}}=\left[\begin{array}{ccc}
0 & 0 & 0 \\
0 & \varepsilon_{2} \mathbf{I} & 0 \\
0 & 0 & \varepsilon_{3} \mathbf{I}
\end{array}\right]
\end{gathered}
$$


with the following differential inequality:

$$
\dot{V}\left(\mathbf{s}_{r}\right) \leq-\alpha_{\varepsilon} V+\beta_{\varepsilon}-2 \beta \mathbf{s}_{r}^{\top} \operatorname{sat}\left(\gamma \mathbf{s}_{r}\right)
$$

where $\beta_{\varepsilon}>0$, then, there exists an invariant ellipsoid for the closed-loop system (5) and (19) in three different cases:

- $\beta=0$ :

$$
\mathcal{E}_{1}=\left\{\mathbf{s}_{r} \in \mathbb{R}^{3} \mid \mathbf{s}_{r}^{\top} \mathbf{J} \mathbf{s}_{r} \leq \frac{\beta_{\varepsilon}}{\alpha_{\varepsilon}}\right\}
$$

- when $\operatorname{sat}\left(\gamma \mathbf{s}_{r}\right)$ is saturated:

$$
\mathcal{E}_{2}=\left\{\mathbf{s}_{r} \in \mathbb{R}^{3} \mid \mathbf{s}_{r}^{\top} \mathbf{J} \mathbf{s}_{r} \leq \frac{\beta_{\varepsilon}-6 \frac{\beta}{\lambda_{\max }(\gamma)}}{\alpha_{\varepsilon}}\right\}
$$

- when $\operatorname{sat}\left(\gamma \mathbf{s}_{r}\right)=\gamma \mathbf{s}_{r}$ :

$$
\mathcal{E}_{3}=\left\{\mathbf{s}_{r} \in \mathbb{R}^{n} \mid \mathbf{s}_{r}^{\top} \mathbf{J} \mathbf{s}_{r} \leq \frac{\beta_{\varepsilon}}{\alpha_{\varepsilon}+\frac{2 \beta \lambda_{\min }(\gamma)}{\lambda_{\max }(\mathbf{J})}}\right\}
$$

which assure practical stability for $\mathbf{s}_{r}$, that is, there exists a time $t_{1}>0$ such that $\left\|\mathbf{s}_{r}\right\| \leq \delta$, where $\delta$ is the upper bound of $\mathbf{s}_{r}$ in the stability region defined by any of the ellipsoids in (20)-(22), $\forall t>t_{1}$, implying that $\exists t_{2} \geq t_{1}$ such that $\left\|\mathbf{q}_{e}\right\| \leq \frac{\delta}{\lambda_{\min }(\boldsymbol{\alpha})}$ and $1 \geq q_{0 e} \geq \sqrt{1-\frac{\delta^{2}}{\lambda_{\min }(\alpha)^{2}}}, \quad \forall t>t_{2}$.

Proof. Consider the following positive-definite energy equation

$$
V=\mathbf{s}_{r}^{\top} \mathbf{J} \mathbf{s}_{r} .
$$

Obtaining the time derivative of (23) it results

$$
\dot{V}=2 \mathbf{s}_{r}^{\top}\left(-\mathbf{K}_{d} \mathbf{s}_{r}-\beta \operatorname{sat}\left(\gamma \mathbf{s}_{r}\right)+\zeta\right)
$$

and using the bounds of (17), (18) and the assumptions in section 3, it follows

$$
\begin{aligned}
& \dot{V}=\left[\begin{array}{c}
\mathbf{s}_{r} \\
\zeta
\end{array}\right]^{\top}\left[\begin{array}{cc}
-\mathbf{K}_{d}-\mathbf{K}_{d}^{\top} & \mathbf{I} \\
\mathbf{I} & 0
\end{array}\right]\left[\begin{array}{c}
\mathbf{s}_{r} \\
\zeta
\end{array}\right]-2 \beta \mathbf{s}_{r}^{\top} \operatorname{sat}\left(\gamma \mathbf{s}_{r}\right) \\
& \pm \alpha_{\varepsilon} V \pm \varepsilon_{1}\|\zeta\|^{2} \pm \varepsilon_{2}\left\|\omega_{e}\right\|^{2} \pm \varepsilon_{3}\left\|\omega_{d}\right\|^{2} \\
& \leq\left[\begin{array}{c}
\mathbf{s}_{r} \\
\zeta
\end{array}\right]^{\top}\left[\begin{array}{cc}
-\mathbf{K}_{d}-\mathbf{K}_{d}^{\top}+\alpha_{\varepsilon} \mathbf{J} & \mathbf{I} \\
\mathbf{I} & -\varepsilon_{1} \mathbf{I}
\end{array}\right]\left[\begin{array}{c}
\mathbf{s}_{r} \\
\zeta
\end{array}\right] \\
& -\alpha_{\varepsilon} V+\varepsilon_{1} c_{2}+\varepsilon_{1} \tilde{\mathbf{x}}^{\top} \mathbf{H} \tilde{\mathbf{x}}+\varepsilon_{2}\left(\left\|\mathbf{s}_{r}\right\|^{2}+\lambda_{\max }(\boldsymbol{\alpha})^{2}-\tilde{\mathbf{x}}^{\top} \tilde{\mathbf{F}} \tilde{\mathbf{x}}\right) \\
& -\varepsilon_{2}\left\|\omega_{e}\right\|^{2} \pm \varepsilon_{3}\left\|\omega_{d}\right\|^{2}-2 \beta \mathbf{s}_{r}^{\top} \operatorname{sat}\left(\gamma \mathbf{s}_{r}\right)
\end{aligned}
$$


then, the following inequality is satisfied

$$
\dot{V} \leq \mathbf{z}^{\top} \mathbf{W}_{0} \mathbf{z}-\alpha_{\varepsilon} V+\beta_{\varepsilon}-2 \beta \mathbf{s}_{r}^{\top} \operatorname{sat}\left(\gamma \mathbf{s}_{r}\right)
$$

where $\mathbf{z}=\left[\tilde{\mathbf{x}}^{\top}, \zeta^{\top}\right]^{\top}, \beta_{\varepsilon}=\varepsilon_{1} c_{2}+\varepsilon_{2} \lambda_{\max }(\boldsymbol{\alpha})^{2}+\varepsilon_{3} \omega_{d}^{+}$. Now, assuming that the set of parameters $\mathbf{K}_{d}, \alpha_{\varepsilon}, \varepsilon_{1}, \varepsilon_{2}, \varepsilon_{3}$ satisfying the LMI $\mathbf{W}_{0} \leq 0$ is not empty, the selection of the "best" parameters that minimize the convergence region, defined by the ellipsoidal matrix $\frac{\beta_{\varepsilon}}{\alpha_{\varepsilon}} \mathbf{J}^{-1}$, can be done using the following optimization problem:

$$
\begin{gathered}
\operatorname{tr}\left\{\frac{\beta_{\varepsilon}}{\alpha_{\varepsilon}} \mathbf{J}^{-1}\right\} \rightarrow \min _{\alpha_{\varepsilon}, \beta_{\varepsilon}, \varepsilon_{1}, \varepsilon_{2}, \varepsilon_{3}, \mathbf{K}_{d}, \mathbf{J}} \\
\text { subject to the constraints } \\
\alpha_{\varepsilon}>0, \varepsilon_{1}>0, \varepsilon_{2}>0, \varepsilon_{3}>0 \\
\mathbf{W}_{0}=\mathbf{W}_{0}\left(\mathbf{K}_{d}, \mathbf{J}, \alpha_{\varepsilon}, \varepsilon_{1}, \varepsilon_{2}, \varepsilon_{3}\right) \leq 0
\end{gathered}
$$

The solution of the optimization problem (25) can be obtained using the MATLAB toolboxes SeDuMi and YALMIP, which effectively use the Interior Point Method [19]. Note that, if there exists a solution that assures $\mathbf{W}_{0} \leq 0$ in (24), then, practical stability for $\mathbf{s}_{r}$ is guaranteed even for a value of $\beta=0$. If $\beta=0$, the ellipsoid is given by (20). Assuming that $\beta>0$, with different values of each component of the vector $\mathbf{s}_{r}$, the saturation function can be in the linear or nonlinear region, then, let us consider the following scenarios:

1. $\left|\gamma_{i} s_{r i}\right|>1$ :

In this region, the saturation function is the same as the sign function, therefore the following inequality is equivalent to (24)

$$
\dot{V} \leq z^{\top} \mathbf{W}_{0} z-\alpha_{\varepsilon} V-2 \beta \mathbf{s}_{r}^{\top} \operatorname{sign}\left(\mathbf{s}_{r}\right)+\beta_{\varepsilon}
$$

where $\operatorname{sign}\left(\mathbf{s}_{r}\right)=\left[\operatorname{sign}\left(s_{r 1}\right), \operatorname{sign}\left(s_{r 2}\right), \operatorname{sign}\left(s_{r 3}\right)\right]^{\top}$. If there is a solution for $\mathbf{W}_{0} \leq$ 0 , then the following inequality is obtained

$$
\dot{V} \leq-\alpha_{\varepsilon} V-2 \beta\left\|\mathbf{s}_{r}\right\|_{1}+\beta_{\varepsilon}
$$

In consequence, exist two cases that depend on the values of $\beta_{\varepsilon}$ and $\left\|\mathbf{s}_{r}\right\|_{1}$ :

(a) $\left\|\mathbf{s}_{r}\right\|_{1} \geq \frac{\beta_{\varepsilon}}{2 \beta}$ :

If this condition is fulfilled, the equation (24) results in

$$
\dot{V} \leq-\alpha_{\varepsilon} V<0
$$

Then, the term $\beta \operatorname{sat}\left(\gamma \mathbf{s}_{r}\right)$ compensates completely the uncertainties bounded by $\beta_{\varepsilon}$, and considering that $-\alpha_{\varepsilon} V$ is negative definite, then $\dot{V}<0$ is guaranteed for $\left\|\mathbf{s}_{r}\right\|_{1} \geq \frac{\beta_{\varepsilon}}{2 \beta}$. Therefore $\exists t_{1}>0$ such that $\left\|\mathbf{s}_{r}\right\|_{1} \leq \frac{\beta_{\varepsilon}}{2 \beta}, \forall t>t_{1}$.

(b) $\left\|\mathbf{s}_{r}\right\|_{1} \leq \frac{\beta_{\varepsilon}}{2 \beta}$ :

Assuming that $\operatorname{sat}\left(\gamma \mathbf{s}_{r}\right)$ is saturated, then each element $\left|s_{r i}\right| \geq \frac{1}{\gamma_{i}}$, resulting in $\left\|\mathbf{s}_{r}\right\|_{1} \geq 3 \bar{\gamma}$, where $\bar{\gamma}=\min \left\{\frac{1}{\gamma_{1}}, \frac{1}{\gamma_{2}}, \frac{1}{\gamma_{3}}\right\}=\frac{1}{\lambda_{\max }(\gamma)}$. Therefore, the inequality (24) becomes

$$
\dot{V} \leq-\alpha_{\varepsilon} V-6 \beta \bar{\gamma}+\beta_{\varepsilon}=-\alpha_{\varepsilon} V+\tilde{\beta}_{\varepsilon}
$$


where $\tilde{\beta}_{\varepsilon}=\beta_{\varepsilon}-6 \beta \bar{\gamma}$, and practical stability is guaranteed for $\mathbf{s}_{r}$ with the ellipsoid defined by (21).

2. $\left|\gamma_{i} s_{r i}\right| \leq 1$ :

In this case $\operatorname{sat}\left(\gamma \mathbf{s}_{r}\right)=\gamma \mathbf{s}_{r}$ and (24) results

$$
\begin{aligned}
\dot{V} & \leq z^{\top} \mathbf{W}_{0} z-\alpha_{\varepsilon} V-2 \beta \mathbf{s}_{r}^{\top} \gamma \mathbf{s}_{r}+\beta_{\varepsilon} \\
& \leq-\alpha_{\varepsilon} V-2 \beta \lambda_{\min }(\boldsymbol{\gamma})\left\|\mathbf{s}_{r}\right\|^{2}+\beta_{\varepsilon}
\end{aligned}
$$

therefore, using $\mathbf{s}_{r}^{\top} \mathbf{J} \mathbf{s}_{r} \leq \lambda_{\max }(\mathbf{J})\left\|\mathbf{s}_{r}\right\|^{2}$, and multiplying it by $-2 \beta \lambda_{\min }(\gamma)$, equals to

$$
\begin{gathered}
-2 \beta \lambda_{\min }(\gamma) \mathbf{s}_{r}^{\top} \mathbf{J} \mathbf{s}_{r} \geq-2 \beta \lambda_{\min }(\gamma) \lambda_{\max }(\mathbf{J})\left\|\mathbf{s}_{r}\right\|^{2} \\
\therefore-\frac{2 \beta \lambda_{\min }(\gamma)}{\lambda_{\max }(\mathbf{J})} \mathbf{s}_{r}^{\top} \mathbf{J} \mathbf{s}_{r} \geq-2 \beta \lambda_{\min }(\gamma)\left\|\mathbf{s}_{r}\right\|^{2}
\end{gathered}
$$

Substituting (27) into (26), the following inequality is obtained

$$
\dot{V} \leq-\left(\alpha_{\varepsilon}+\frac{2 \beta \lambda_{\min }(\gamma)}{\lambda_{\max }(\mathbf{J})}\right) V+\beta_{\varepsilon}
$$

Consequently, practical stability is assured with the ellipsoid defined by (22)

Note that in each case, the saturation term in the control law helps to reduce the effects produced by disturbances and uncertainties in the dynamic system represented by $\beta_{\epsilon}$ in (24). This reduces the region of the "original ellipsoid" (20) in every case: (21) and (22). With this result in mind, let us analyze the attitude error stability using the following positive-definite function

$$
V_{q}=\left(1-q_{0 e}\right)^{2}+\mathbf{q}_{e}^{\top} \mathbf{q}_{e}
$$

getting the time derivative, and assuming that $\mathbf{s}_{r}$ has reached its bound $\delta$, from (16) the following expression can be obtained

$$
\dot{V}_{q}=\mathbf{q}_{e}^{\top} \omega_{e} \leq-\left(\lambda_{\min }(\boldsymbol{\alpha})\left\|\mathbf{q}_{e}\right\|-\delta\right)\left\|\mathbf{q}_{e}\right\|
$$

therefore if $\left\|\mathbf{q}_{e}\right\|>\frac{\delta}{\lambda_{\min }(\boldsymbol{\alpha})}$, then it is assured that $\dot{V}_{q}<0$. Then $\exists t_{2} \geq t_{1}$ such that $\left\|\mathbf{q}_{e}\right\| \leq \frac{\delta}{\lambda_{\min }(\boldsymbol{\alpha})} \forall t>t_{2}$. Using the restriction of the unit quaternion, the maximum bound of the scalar part of the quaternion $q_{0 e}$ can be estimated as follows:

$$
q_{0 e}^{2}+\mathbf{q}_{e}^{\top} \mathbf{q}_{e}=1, \quad 1 \geq q_{0 e} \geq \sqrt{1-\frac{\delta^{2}}{\lambda_{\min }(\boldsymbol{\alpha})^{2}}}, \quad \forall t>t_{2} .
$$

\section{Numerical Results}

The performance of the controller is tested using the saturation term:

$$
\tanh \left(\gamma \mathbf{s}_{r}\right)=\left[\tanh \left(\gamma_{1} s_{r 1}\right), \tanh \left(\gamma_{2} s_{r 1}\right), \tanh \left(\gamma_{3} s_{r 1}\right)\right]^{\top}
$$




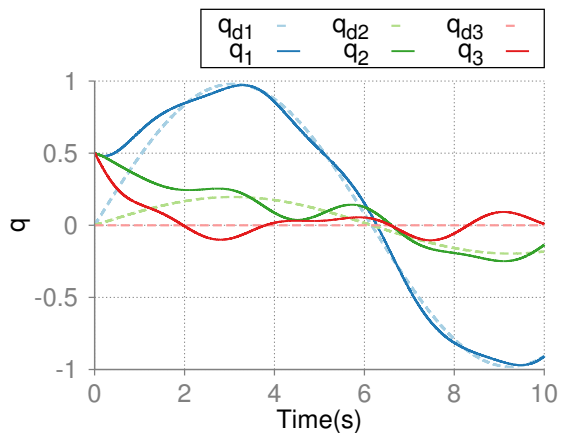

(a) Quaternion response without saturation term.

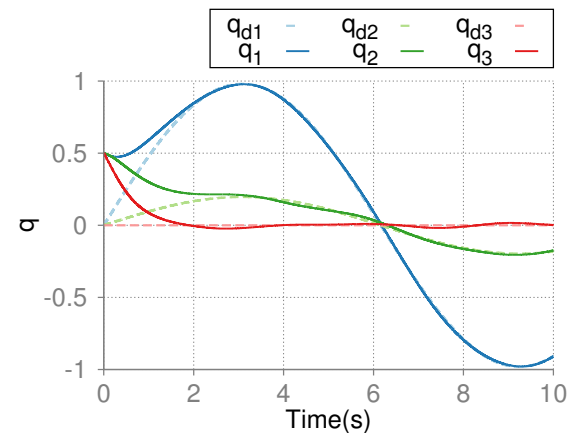

(b) Quaternion response with saturation term $\tanh \left(\gamma \mathbf{s}_{r}\right)$.

Figure 2: Comparative of the signals between the system with and without the saturation $\operatorname{term} \tanh \left(\gamma \mathbf{s}_{r}\right)$.

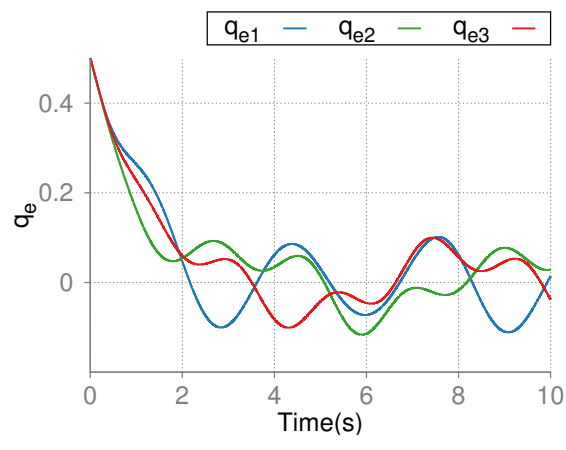

(a) Error quaternion response.

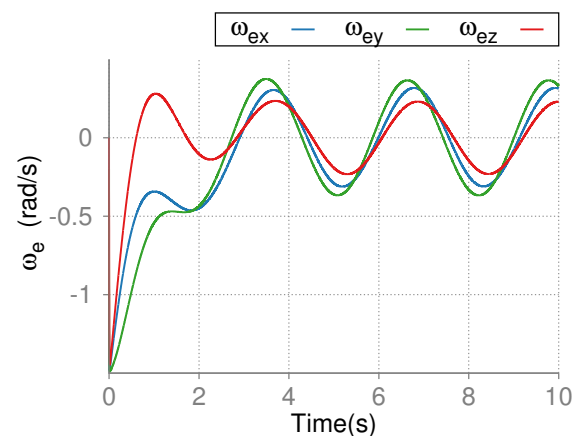

(b) Error angular rate response.

Figure 3: Error signals without saturation term, or $\beta=0$.

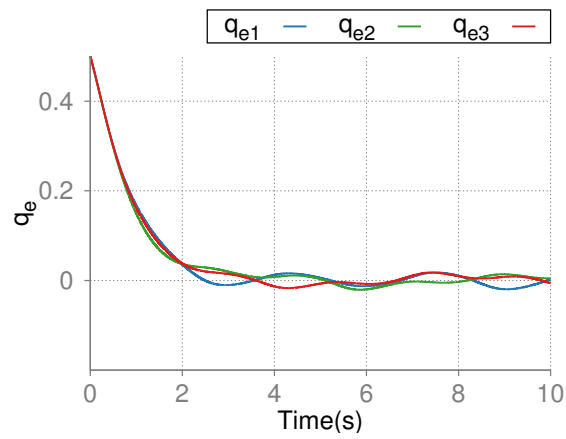

(a) Error quaternion response.

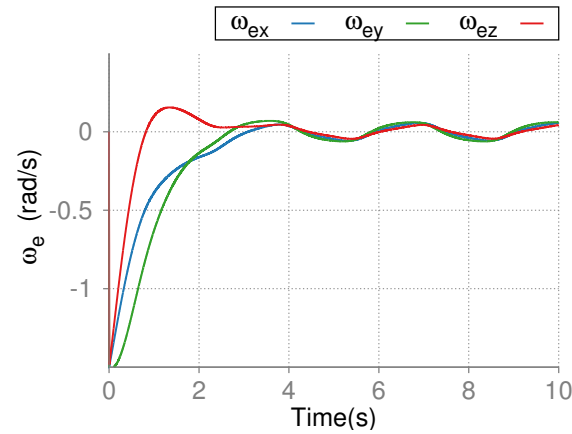

(b) Error angular rate response.

Figure 4: Error signals with saturation term $\tanh \left(\gamma \mathbf{s}_{r}\right)$. 


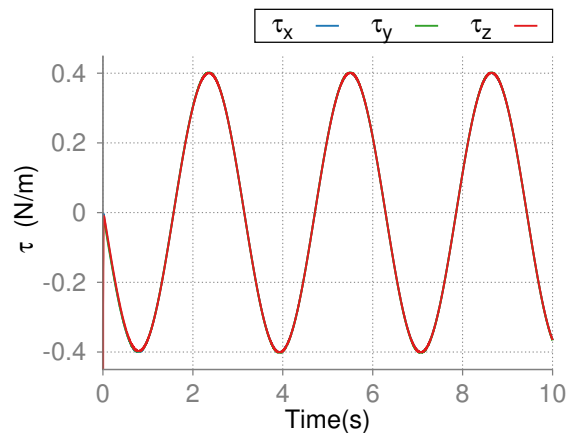

(a) Torques without using the saturation term.

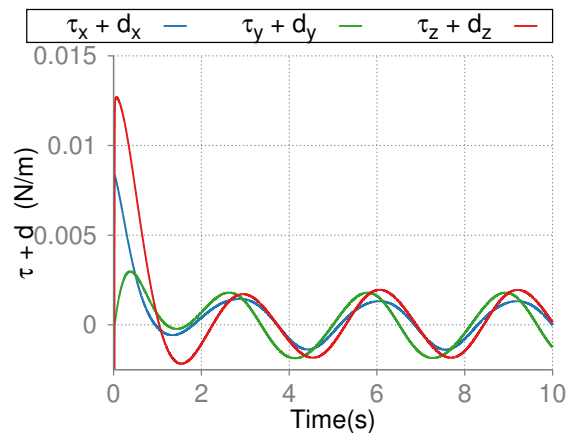

(c) Sum of torques and distrubances without using (d) Sum of torques and disturbances with $\tanh \left(\gamma \mathbf{s}_{r}\right.$ ). the saturation term.

Figure 5: Comparative of the torques with and without saturation term.
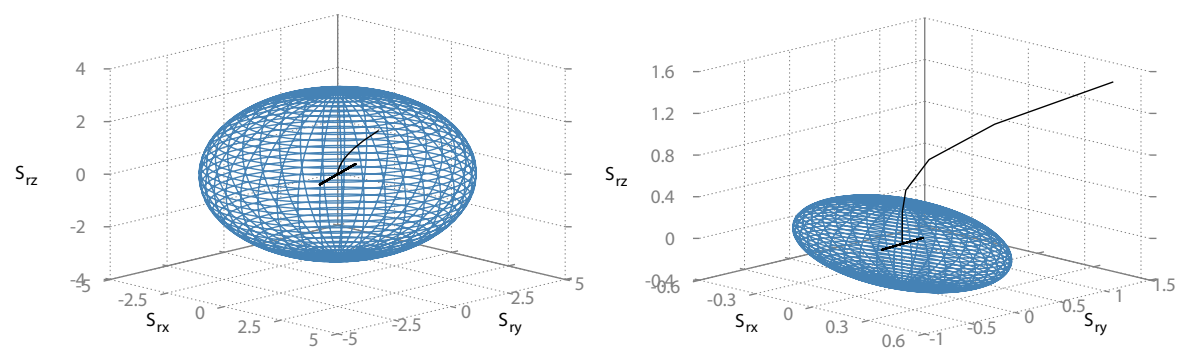

(a) $\mathbf{s}_{r}$ response with the estimated region of attrac- (b) $\mathbf{s}_{r}$ response with the estimated region of attraction without using the saturation term. tion with $\tanh \left(\gamma \mathbf{s}_{r}\right)$

Figure 6: Comparative of the $\mathbf{s}_{r}$ responses with estimated regions of attraction with and without saturation term.

where $\tanh \left(\gamma_{i} s_{r i}\right)$ is a smooth function, for $i=1,2,3$. Also, a comparative test without using the saturation term is presented in order to demonstrate the effect of the nonlinear term on the closed-loop system.

The simulation was performed in the program MATLAB ${ }^{\circledR}$ Simulink ${ }^{\circledR} 8.3$, with a fixed 


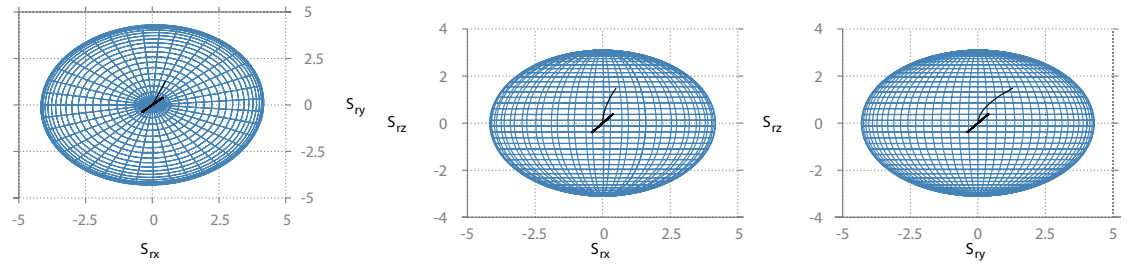

(a) Ellipsoid without using the (b) Ellipsoid without using the (c) Ellipsoid without using the saturation term in the $x y$ plane. saturation term in the $x z$ plane. saturation term in the $y z$ plane.

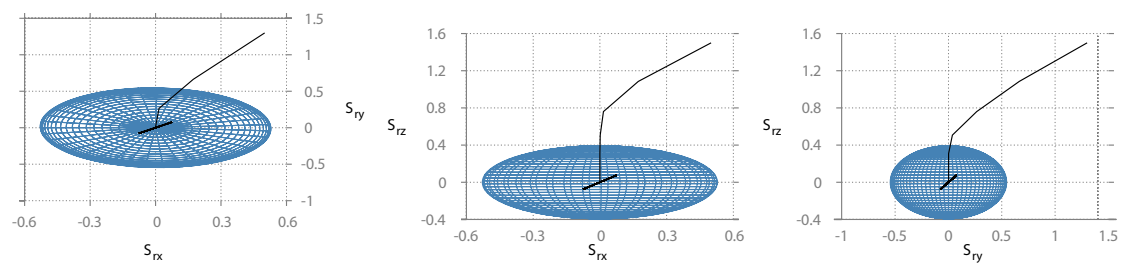

(d) Ellipsoid with $\tanh \left(\gamma \mathbf{s}_{r}\right)$ in the (e) Ellipsoid with $\tanh \left(\gamma \mathbf{s}_{r}\right)$ in (f) Ellipsoid with $\tanh \left(\gamma \mathbf{s}_{r}\right)$ in $x y$ plane. the $x z$ plane. the $y z$ plane.

Figure 7: Comparative of the $\mathbf{s}_{r}$ responses with estimated regions of attraction, projections in the $x y, x z$ and $y z$, with and without saturation term.

step of $0.001 s$ and solver Dormand-Price (ode8). The simulation parameters are: the disturbance function is denoted by $\mathbf{d}(t)=0.4 \sin (2 t)[1,1,1]^{\top} \mathrm{Nm}$, the desired trajectory for the angular velocity is $\omega_{d}=[1,0.2,0]^{\top} \mathrm{rad} / \mathrm{s}$, the desired quaternion $\overline{\mathbf{q}}_{d}$ is calculated using equation (7) with initial condition $\overline{\mathbf{q}}_{d}(0)=[1,0,0,0]^{\top}$. The initial conditions of the system are $\overline{\mathbf{q}}(0)=[0.5,0.5,0.5,0.5]^{\top}$ and $\omega(0)=[0,0,0]^{\top} \mathrm{rad} / \mathrm{s}$, the gains are $\mathbf{K}_{d}=\operatorname{diag}(1,1,1), \alpha=\operatorname{diag}(6,6,6), \beta=0.4, \gamma=\operatorname{diag}(15,15,15)$ and the inertia matrix is given by:

$$
\mathbf{J}=10^{-6}\left[\begin{array}{ccc}
2.098\left(10^{3}\right) & 63.577538 & -2.002648 \\
63.577538 & 2.102\left(10^{3}\right) & 0.286186 \\
-2.002648 & 0.286186 & 4.068\left(10^{3}\right)
\end{array}\right]
$$

With these parameters, the solution for the practical stability is: $\alpha_{\varepsilon}=35.989, \varepsilon_{1}=$ $0.583, \varepsilon_{2}=8.26 \times 10^{-4}, \varepsilon_{3}=1 \times 10^{-4}, \beta_{\varepsilon}=1.4069$, with the parameters $c_{0}=0, c_{1}=$ $1.6810 \times 10^{-5}, d^{+}=0.4800, \omega_{d}^{+}=1.04, \dot{\omega}^{+}=0$. The results using $\beta=0$ and $\beta=0.4$ (without and with the saturation term) are shown in Figures 2. In Figure 2(a) the system tries to reach the desired attitude, but the disturbance causes poor performance of the tracking, producing important oscillations in the error signals, as can be seen in Figures 3(a) and 3(b). However, using the saturation term the performance of the tracking is improved, which is shown in Figure 2(b). Also, the amplitudes of the error signals are reduced, see Figures 4(a) and 4(b).

It is clear how the attitude trajectory tracking is improved by using the saturation term. The control torques are shown in Figures 5(a) and 5(b). Notably, it can be seen that the improved performance with the saturation term is achieved with practically the same control magnitude. Additionally, resultant torques, which are the sum of torques and disturbances, are shown in Figures 5(c) and 5(d), for the sake of clarity. It can be seen 
that the magnitude of the resultant torque with the saturation term is smaller than the one without it. Even with this magnitude, a better compensation is obtained thanks to the fast response of the saturation term. Note that the slight difference between the torques generates remarkable differences in the angular rate response. This is due to the fact that the quadrotor has a small inertia, which can be observed when $\dot{\mathbf{s}}_{r}$ is solved from equation (13). Also, the region of convergence is different in the two cases. In Figure 6(a), the ellipsoid with $\beta=0$, given by (20), is shown. Figure 6(b) presents the ellipsoid of the region of convergence using the saturation term, given by (22). From Figure 6(b), it is clear that the saturation term reduces the effects of uncertainties and the external disturbances, allowing the error trajectories to be confined to a small ellipsoid. In Figure 7, the projected ellipsoids on the planes $x y, x z$ and $y z$ are shown. It can be easily seen the differences between regions of convergence.

\section{Experimental Results}

Well performance of the attitude control is demonstrated when the aerial vehicle executes an aggressive trajectory (flips) about the $y$ axis. The experiments were performed in both indoor and outdoor environments. The platform used is an AR Drone 2.0 Edition of Parrot.

\subsection{Experimental Setup}

The platform includes an embedded system with an ARM Cortex A8 $1 \mathrm{GHz}$ processor with $1 \mathrm{~GB}$ of RAM. The control algorithms are programmed in $\mathrm{C}++$ code using Codeblocks 13 in a Linux Ubuntu 14.04 environment. The IMU measurements and the control torques are updated at $200 \mathrm{~Hz}(5 \mathrm{~ms})$.

The thrust $T$ is used to control the altitude of the quadrotor by using the information of an altitude ultrasonic sensor to compute an altitude PD controller with compensation of gravity, where its gain parameters are $K_{p z}=0.6$ and $K_{v z}=0.45$. The computed control inputs, $\tau$ and $T$, are mapped to desired angular velocities that are then passed to the rotors' drivers, see the diagram in Figure 8.

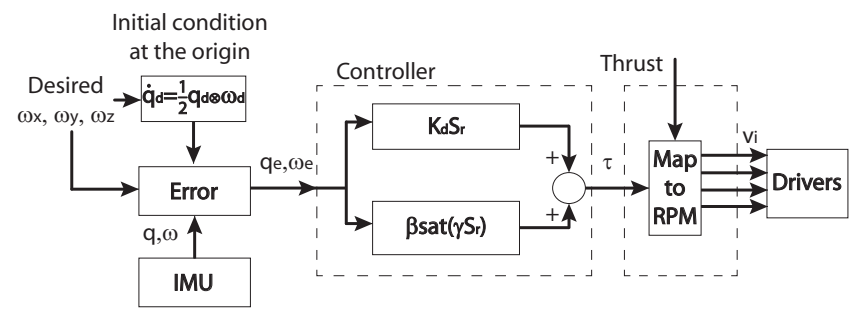

Figure 8: Diagram of the input signals in the vehicle.

In order to allow the vehicle to have enough altitude to perform the aggressive maneuver, four stages in the attitude control of the vehicle are included [10], see Figure 9: 
1.- The first stage involves a maximum thrust to obtain an upward acceleration during the tracking of the trajectory. There is a predefined time, given by the user, for this phase. The larger the number of flips, the larger the altitude needed to avoid a crash.

2.- In the second stage, there is no attempt to control the altitude of the vehicle, only to follow in closed loop the desired attitude trajectory. The time of this phase depends on the number of flips, the maximum angular velocity, and the acceleration for the desired trajectory, and can be decreased if the maximum angular velocity is incremented. Also, the thrust is regulated to a constant value while the vehicle performs the flips. In this stage the vehicle reaches its maximum altitude (from the first stage) while performing the maneuver, then, the vehicle begins to fall as a result of gravity.

3.- At the third stage, after the maneuver is performed, the signal of the maximum thrust is given to compensate the gravitational acceleration and stop the vehicle from falling.The duration of this phase is given by the user and also depends on the number of the flips performed, as in the first stage. The larger the number of flips, the faster the fall of the vehicle, consequently more time is needed.

4.- The final stage is to regulate the altitude to a desired point. The duration of this phase ends until another sequence is started.

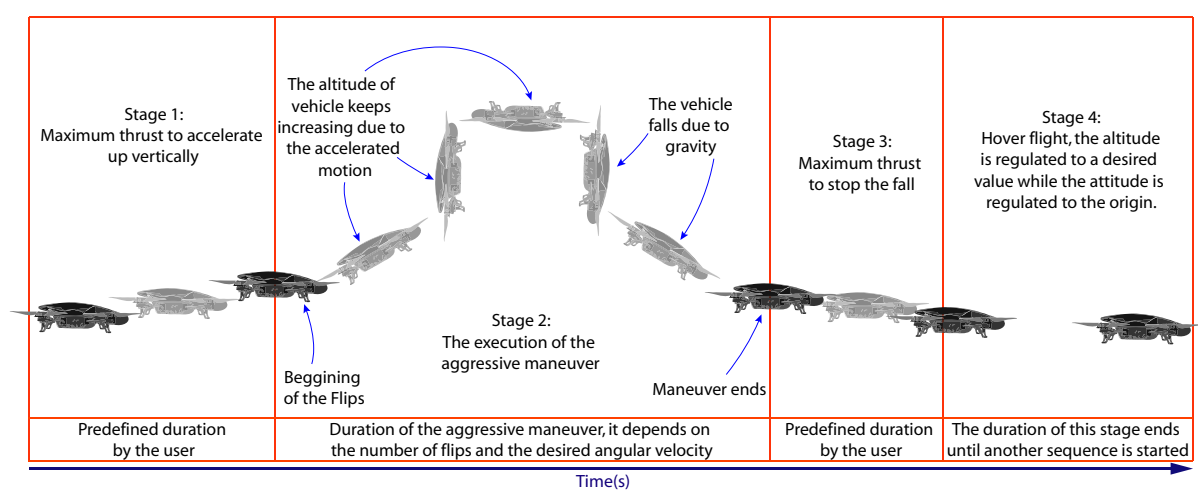

Figure 9: Diagram of the stages while performing an aggressive maneuver.

The desired trajectory for the angular velocity is $\omega_{d}=\left[0, \omega_{d y}(t), 0\right]^{\top}$, where $\omega_{d y}(t)$ is a trapezoidal function, with maximum value of $1400 \mathrm{deg} / \mathrm{s}$ and the desired acceleration of $\pm 5000 \mathrm{deg} / s^{2}$ at the beginning and the end of the trajectory.

The desired quaternion is $\overline{\mathbf{q}}_{d}=\left[\cos \left(\theta_{d} / 2\right), 0, \sin \left(\theta_{d} / 2\right), 0\right]^{\top}$, where $\theta_{d}$ is calculated resolving the equation $\dot{\theta}_{d}(t)=\omega_{d t}(t)$ assuming initial condition $\theta_{d}(0)=0$, and the hyperbolic tangent function is used as saturation. The solution for the practical stability problem was found with the following parameters: $\mathbf{K}_{d}=\operatorname{diag}(0.35,0.45,0.7), \alpha_{\varepsilon}=$ 1.504, $\varepsilon_{1}=1.3199, \varepsilon_{2}=9.9 \times 10^{-2}, \varepsilon_{3}=2 \times 10^{-4}, \beta_{\varepsilon}=45.9545$, with $c_{0}=0, c_{1}=$ $2 \times 10^{-5}, d^{+}=3, \omega_{d}^{+}=24.5^{2}, \dot{\omega}^{+}=87.26^{2}$ and the gains $\alpha=\operatorname{diag}(11.43,8.88,5.71)$, 


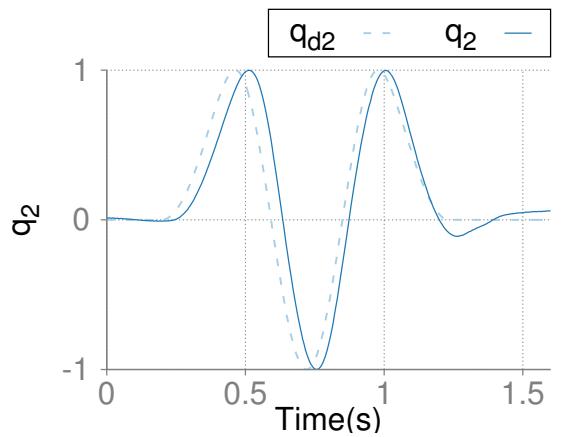

(a) Quaternion response in real time.

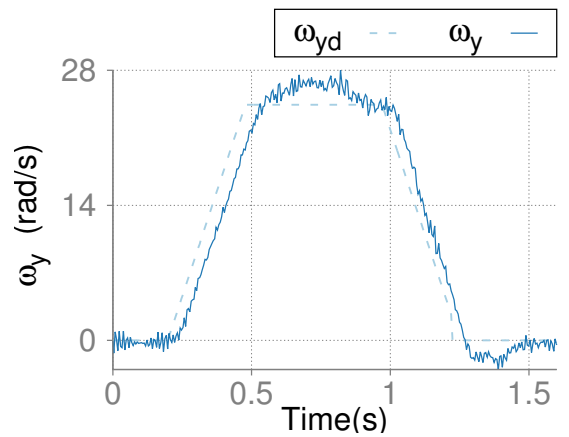

(b) Angular rate response in flight test.

Figure 10: Desired and actual signals of the vehicle during the tracking of 3 flips.

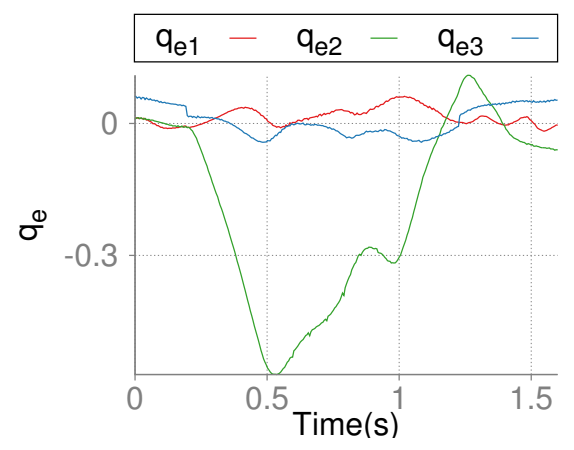

(a) Error quaternion behavior.

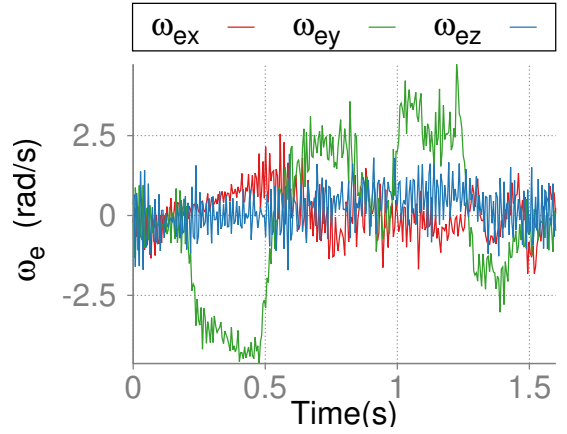

(b) Error angular rate performance.

Figure 11: State signals of the vehicle during the tracking of 3 flips.

$\beta=0.5, \gamma=\operatorname{diag}(0.245,0.315,0.49)$, using an approximation of the inertia matrix $\mathbf{J}=$ $\operatorname{diag}(0.002237568,0.002985236,0.00480374)$ for solving the optimization problem.

\subsection{Indoor results}

The experimental tests were carried out for one, two, and three flips as aggressive desired trajectories, but only the result for the execution of 3 flips is presented as the most representative experiment. The results of the system performing the flips are presented in the Figures 10,11 and 12(a). The desired trajectory is fast and aggressive, lasted about a second. Even with this challenging trajectory, the vehicle follows the desired trajectory as can be seen in Figures 10(a) and 11(b).

Also, the quaternion error has its maximum value in the $q_{e 2}$ component only at the beginning of the multi-flips trajectory, but as time increases the error decreases, as shown in Figure 10(a). The same behavior applies for the angular velocity error, see Figure 10(b). The largest component in the control input is $\tau_{y}$ as a result of the desired trajectory where the most demanding torque is the $y$ axis, as can be seen in Figure 

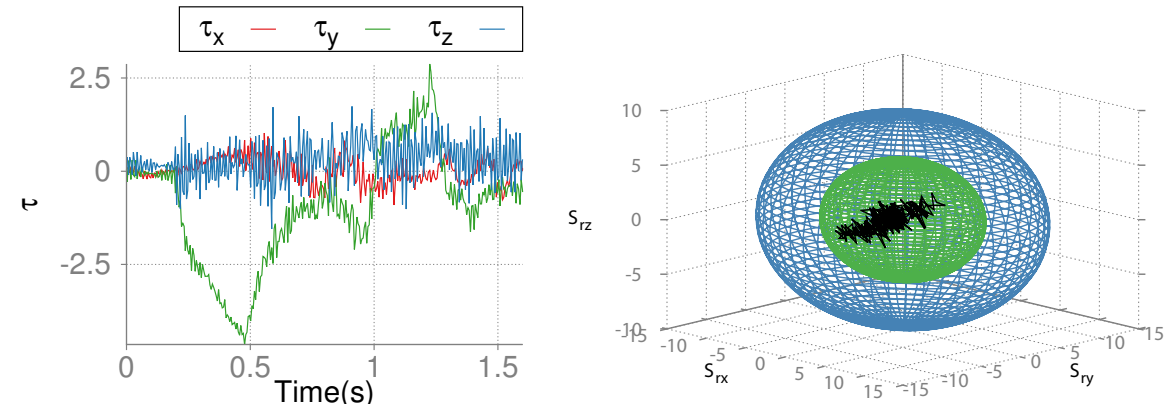

(a) Control torque signal for the vehicle during (b) 3D view of the ellipsoid of the region of attraction the tracking of 3 flips. for $\mathbf{s}_{r}$.
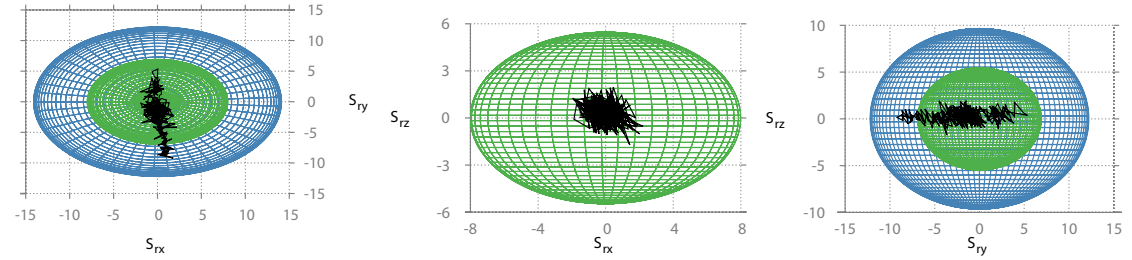

(c) $\mathrm{XY}$ view of the ellipsoid of (d) XZ view of the ellipsoid of (e) YZ view of the ellipsoid of the region of attraction for $\mathbf{s}_{r}$. the region of attraction for $\mathbf{s}_{r}$. the region of attraction for $\mathbf{s}_{r}$.

Figure 12: Torque and $\mathbf{s}_{r}$ response with estimated region of attraction in the experimental results of 3 flips.

12(a). The ellipsoid of the convergence region is shown in Figure 12, which has a size that depends on the aggressive trajectory reflected in the value of $\beta_{\varepsilon}$.

In Figure 12(b) two ellipsoids are present, the bigger one corresponds to $\mathcal{E}_{2},(21)$, (the saturated region), and the smaller one ellipsoid corresponds to $\mathcal{E}_{3},(22)$, (the linear region of the saturation term). As long as $\operatorname{sat}\left(\gamma \mathbf{s}_{r}\right)=\gamma \mathbf{s}_{r}$, the trajectories of $\mathbf{s}_{r}$ stay in the green ellipsoid, (22), this is the case when the disturbances and uncertainties of the system are smaller than $\beta$, that is, $\beta>\beta_{\varepsilon}$. When $\beta_{\varepsilon}>\beta$, the trajectories of $\mathbf{s}_{r}$ would leave the ellipsoid (22), but remain in the ellipsoid (21). In fact, this scenario is present in the experiments, as can be seen in Figures 12(c) and 12(e) the system leaves the green ellipsoid (22) only in the $y$ axis, where the tracking of the aggressive maneuver is performed. For the $x$ and $z$ axes, the system remains in the ellipsoid (22), see Figure $12(d)$.

\subsection{Outdoor results}

Figures 13-16 show the successful experimental results of 2 flips outdoors. The performance of the controller is slightly affected by the environment conditions, as can be seen in Figures 14 and 15. In fact, the attitude error is qualitatively similar to that shown in Figure 11, however, it is quantitatively greater than the attitude error of the indoor test. From Figure 14, it can be observed that the phase difference affects the performance of the attitude error, and thus, the performance of the controller, too, see Figure 16(a). The ellipsoids in both indoor and outdoor tests are the same since the 


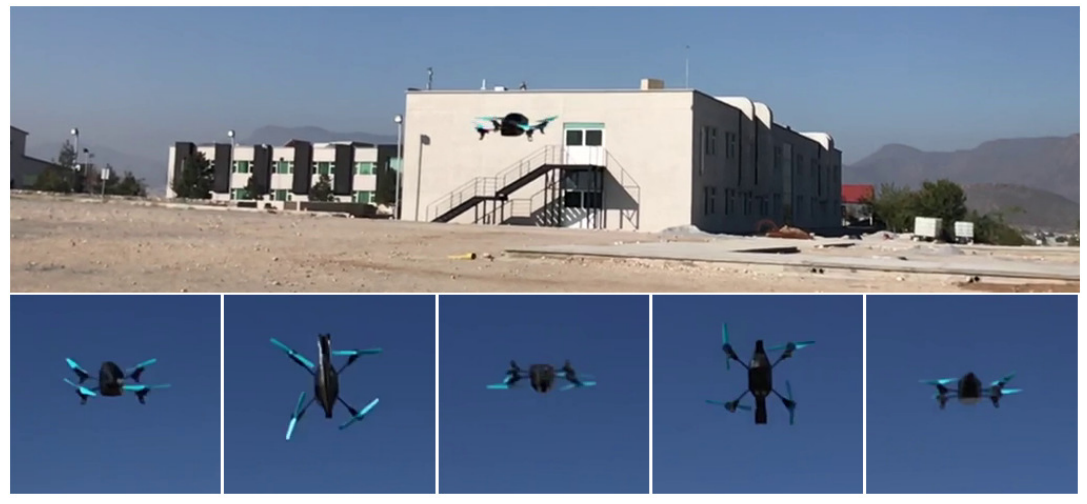

Figure 13: Outdoors experiments of the vehicle performing flips.

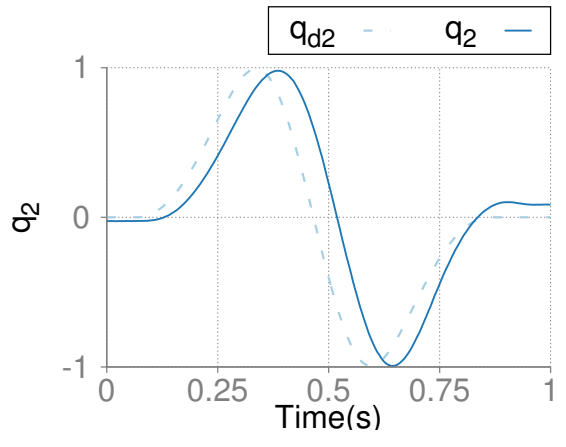

(a) Quaternion response in real time.

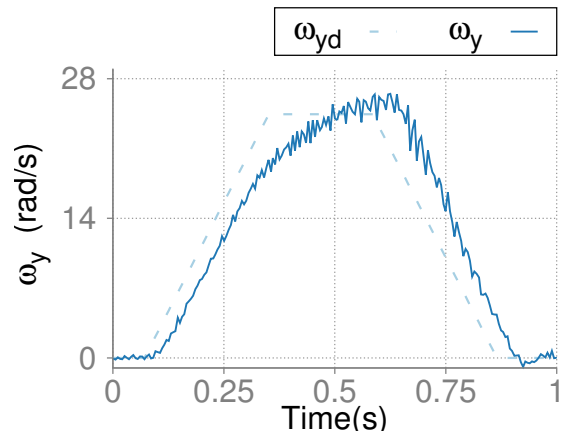

(b) Angular rate response in flight test.

Figure 14: Desired and actual signals of the vehicle during the tracking of 2 flips outdoors.

same control parameters are used, however, outdoor disturbances, such as gust, have an effect on the trajectories of $\mathbf{s}_{r}$, as can be seen in Figures 16(c) and 16(e).

The video of the experiments can be accessed in https://www.youtube. $\mathrm{com} /$ watch?v=gUOa98wrMeo where the results demonstrate the viability of the proposed approach. The outdoor experiments include one and two flips, some frames can be seen in Figure 13.

\subsection{Implementation Considerations}

The control algorithm is implemented directly on the quadrotor processing system by considering only the discrete-time attitude measurements. It is executed with a sampling period of $5 \mathrm{~ms}$, which is small enough to ensure that the plant response approximates the response of the continuous system. However, control parameters were tuned according to the latency issues in the sense that only relatively small gains from the LMI solution were considered. Also, the saturation term has to be tuned to ensure that the controller does not induce high frequency signals, which may cause instability. 


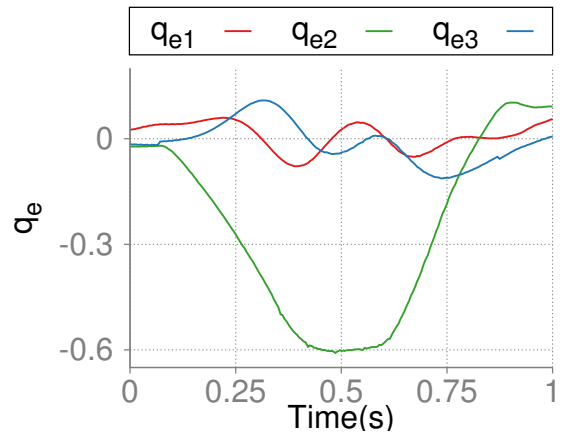

(a) Error quaternion behavior.

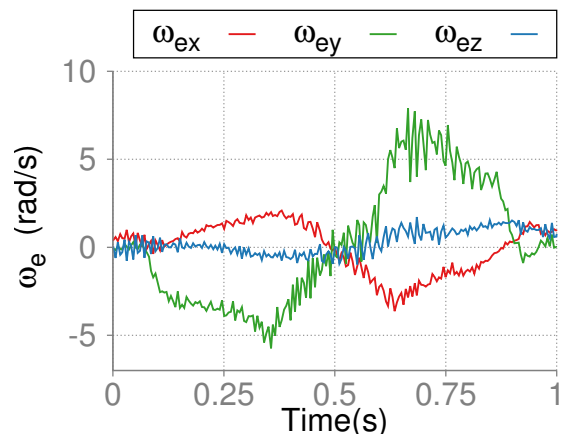

(b) Error angular rate performance.

Figure 15: State signals of the vehicle during the tracking of 2 flips outdoors.
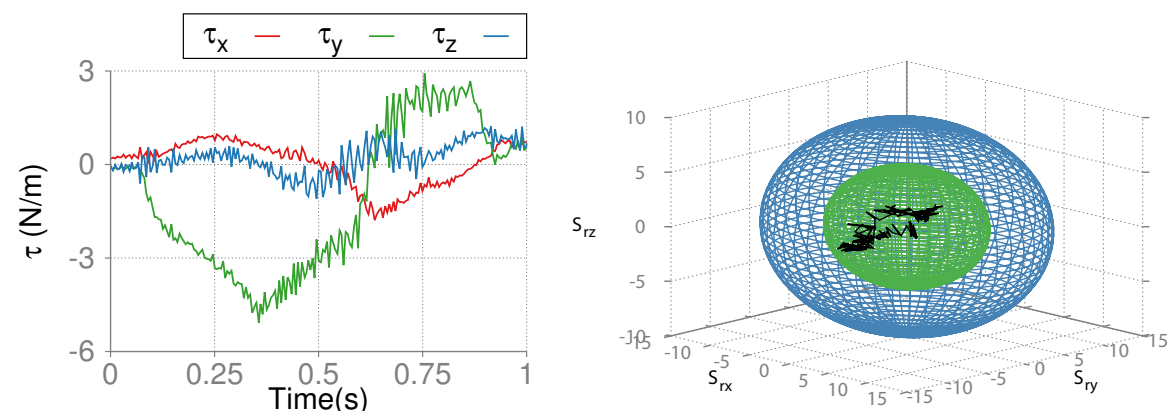

(a) Control torque signal for the vehicle during (b) 3D view of the ellipsoid of the region of attraction the tracking of 2 flips outdoors. for $\mathbf{s}_{r}$.
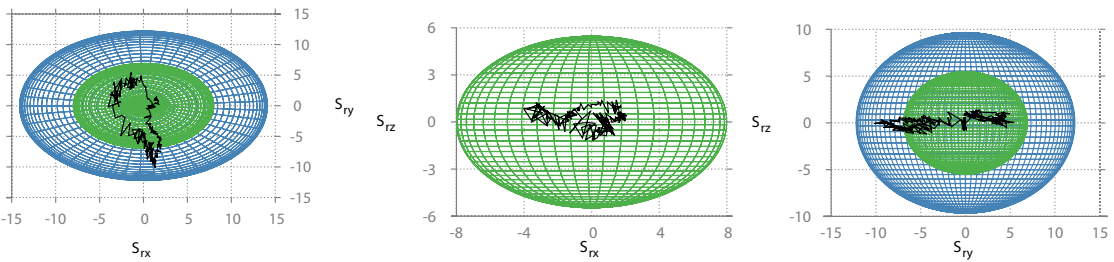

(c) XY view of the ellipsoid of (d) XZ view of the ellipsoid of (e) YZ view of the ellipsoid of the region of attraction for $\mathbf{s}_{r}$. the region of attraction for $\mathbf{s}_{r}$. the region of attraction for $\mathbf{s}_{r}$.

Figure 16: Torque and $\mathbf{s}_{r}$ response with estimated region of attraction of the variable $\mathbf{s}_{r}$ in the experimental results of 2 flips outdoors.

The feasibility of the LMI depends on both the system parameters and the desired angular velocity. Specifically, it depends on the parameters of the inertia matrix $\mathbf{J}$ and upper bounds $\omega_{d}^{+}, \dot{\omega}_{d}^{+}$and $d^{+}$. Large values of these upper bounds increase the upper bound of the ellipsoid $\left(\beta_{\varepsilon}\right.$ depends on $\omega_{d}$ and $\dot{\omega}_{d}$ ) and also could lead to an infeasible $\mathbf{W}_{0}$. If the resulting upper bounds with a different desired maneuver are lower than or equal to the ones presented in the section 7.1, then the LMI is feasible and the com- 
puted controller parameters can be directly implemented, otherwise the feasibility of the LMI needs to be verified.

In order to estimate the gain $\mathbf{K}_{d}$ and the region of the attractive ellipsoid, a conservative approximation of the matrix $\mathbf{J}$ is used. In fact, the error that results from this approximation is considered as parametric uncertainties, which are included in the disturbance $\zeta$. However, our approach considers conservative bounds of disturbances and uncertainties that correspond to the worst case scenario. If these bounds are not adequately chosen, large gains of $\mathbf{K}_{d}$ may be obtained from the solution of the LMI, which cannot be implemented on the vehicle due to latency.

Unit quaternions have some issues to represent the attitude of the quadrotor, specifically sign ambiguity, which is due to the fact that $\overline{\mathbf{q}}$ represents the same rotation as $-\overline{\mathbf{q}}$. This condition can be present if the desired reference quaternion changes to minus its value suddenly, that is, if at a given instant $t_{p}$ the desired reference is $\overline{\mathbf{q}}_{d}$, then it changes to $-\overline{\mathbf{q}}_{d}$ at $t>t_{p}$. In fact, this was an implementation issue when the desired hover reference is set to $\overline{\mathbf{q}}_{d}=[1,0,0,0]$, and after performing a flip, or any odd number of flips, the desired quaternion ends at $\overline{\mathbf{q}}_{d}\left(t_{p}\right)=[-1,0,0,0]$, resulting in an abrupt transition of the vehicle to reach the desired hover reference $\overline{\mathbf{q}}_{d}=[1,0,0,0]$ for $t>t_{p}$ (another flip without tracking a trajectory since the desired reference is constant). A signal conditioning stage has been implemented to avoid this issue by multiplying the desired reference quaternion by -1 at the end of an odd number of flips.

\section{Conclusions}

A robust nonlinear control algorithm using the combination of the Ellipsoid method and a saturation term applied to a quadrotor is proposed. The proposed approach is free of singularities and ensures a continuous yet robust controller to enforce practical stability of tracking errors, even if the required trajectory is aggressive. It was proven that a state feedback $\mathbf{K}_{d} \mathbf{s} r$ induces an attractive invariant set around the origin, while a saturation term $\beta \operatorname{sat}\left(\gamma \mathbf{s}_{r}\right)$ improves robustness as well as frequency response, attenuating the effects of uncertainties and disturbances with a fast response of the controller, but without compromising its regularity (control signals with frequencies that can be handled by the rotors). The control parameters are obtained by solving an optimization problem in order to reduce as much as possible the region of an attractive ellipsoid in which the attitude error trajectories are confined. The main advantage of the proposed approach is the tracking of aggressive trajectories in a quadrotor system with a continuous closed-loop controller that has robustness properties and is independent of the parameters and dynamical structure of the system. Numerical and experimental data were presented to demonstrate the viability of the proposed scheme. The experiments were performed in both indoor and outdoor environments in which high angular velocity multiple flips were achieved. The tracking is not limited only to this desired aggressive maneuver. Other desired trajectories, such as multiple flips in any other direction, can be implemented, as long as the condition of $\mathbf{W}_{0} \leq 0$ is satisfied. 


\section{Acknowledgments}

Authors acknowledge support from Conacyt of Mexico, under Research Grants 264513 and PhD Scholarship 377697. This work has been sponsored by the French government research programm Investissements d'avenir through the Robotex Equipment of Excellence (ANR-10-EQPX-44), as well as by the framework of the Labex MS2T, funded by the French Government, through the program Investments for the future managed by the National Agency for Research (Reference ANR-11-IDEX-000402).

\section{References}

[1] E. Zheng and J. Xiong, "Quad-rotor unmanned helicopter control via novel robust terminal sliding mode controller and under-actuated system sliding mode controller," Optik - International Journal for Light and Electron Optics, vol. 125, no. 12, pp. $2817-2825,2014$.

[2] H. Liu, D. Li, Z. Zuo, and Y. Zhong, "Robust attitude control for quadrotors with input time delays," Control Engineering Practice, vol. 58, pp. 142 - 149, 2017.

[3] A. Tayebi and S. McGilvray, "Attitude stabilization of a vtol quadrotor aircraft," IEEE Transactions on Control Systems Technology, vol. 14, pp. 562-571, May 2006.

[4] J. Guerrero-Castellanos, N. Marchand, A. Hably, S. Lesecq, and J. Delamare, "Bounded attitude control of rigid bodies: Real-time experimentation to a quadrotor mini-helicopter," Control Engineering Practice, vol. 19, no. 8, pp. 790 - 797, 2011.

[5] E. Fresk and G. Nikolakopoulos, "Full quaternion based attitude control for a quadrotor," in 2013 European Control Conference (ECC), pp. 3864-3869, July 2013.

[6] F. Goodarzi, D. Lee, and T. Lee, "Geometric nonlinear pid control of a quadrotor uav on se(3)," in 2013 European Control Conference (ECC), pp. 3845-3850, July 2013.

[7] D. Mellinger, N. Michael, and V. Kumar, "Trajectory generation and control for precise aggressive maneuvers with quadrotors," The International Journal of Robotics Research, vol. 31, pp. 664-674, Apr. 2012.

[8] H. Huang, G. M. Hoffmann, S. L. Waslander, and C. J. Tomlin, "Aerodynamics and control of autonomous quadrotor helicopters in aggressive maneuvering," in 2009 IEEE International Conference on Robotics and Automation, pp. 32773282, May 2009.

[9] M. Furci, G. Casadei, R. Naldi, R. G. Sanfelice, and L. Marconi, "An open-source architecture for control and coordination of a swarm of micro-quadrotors," in 2015 International Conference on Unmanned Aircraft Systems (ICUAS), pp. 139146, June 2015. 
[10] S. Lupashin, A. Schölig, M. Sherback, and R. D’Andrea, "A simple learning strategy for high-speed quadrocopter multi-flips," in Robotics and Automation (ICRA), 2010 IEEE International Conference on, pp. 1642-1648, May 2010.

[11] Y. Chen and N. O. Pérez-Arancibia, "Generation and real-time implementation of high-speed controlled maneuvers using an autonomous 19-gram quadrotor," in 2016 IEEE International Conference on Robotics and Automation (ICRA), pp. 3204-3211, May 2016.

[12] A. A. El-Badawy and M. A. Bakr, "Quadcopter aggressive maneuvers along singular configurations: An energy-quaternion based approach," J. Control Sci. Eng., vol. 2016, pp. 4:4-4:4, Jan. 2016.

[13] L. Wang and J. Su, "Switching control of attitude tracking on a quadrotor uav for large-angle rotational maneuvers," in 2014 IEEE International Conference on Robotics and Automation (ICRA), pp. 2907-2912, May 2014.

[14] M. K. Shaik and J. F. Whidborne, "Robust sliding mode control of a quadrotor," in 2016 UKACC 11th International Conference on Control (CONTROL), pp. 1-6, Aug 2016.

[15] S. Gonzalez-Garcia, A. Polyakov, and A. Poznyak, "Output linear controller for a class of nonlinear systems using the invariant ellipsoid technique," in American Control Conference, 2009. ACC '09., pp. 1160-1165, June 2009.

[16] A. Poznyak, A. Polyakov, and V. Azhmyakov, Attractive Ellipsoids in Robust Control. Springer, 2014.

[17] H. Alazki and A. Poznyak, "Robust output stabilization for a class of nonlinear uncertain stochastic systems under multiplicative and additive noises: The attractive ellipsoid method," Journal of Industrial and Management Optimization, vol. 12, no. 1, pp. 169-186, 2016.

[18] A. Sanchez-Orta, V. Parra-Vega, C. Izaguirre-Espinosa, and O. Garcia, "Positionyaw tracking of quadrotors," Journal of Dynamic Systems, Measurement, and Control, vol. 137, pp. 061011-061011-12, June 2015.

[19] Y. Nesterov, A. Nemirovskii, and Y. Ye, Interior-point polynomial algorithms in convex programming, vol. 13. SIAM, 1994. 